isid/ms/2011/10

August 4, 2011

http://www.isid.ac.in/statmath/eprints

\title{
On a conjecture on Ramanujan primes
}

\author{
Shanta LAISHRAM
}

Indian Statistical Institute, Delhi Centre 7, SJSS Marg, New Delhi-110 016, India 



\title{
ON A CONJECTURE ON RAMANUJAN PRIMES
}

\author{
SHANTA LAISHRAM
}

\begin{abstract}
For $n \geq 1$, the $n$th Ramanujan prime is defined to be the smallest positive integer $R_{n}$ with the property that if $x \geq R_{n}$, then $\pi(x)-\pi\left(\frac{x}{2}\right) \geq n$ where $\pi(\nu)$ is the number of primes not exceeding $\nu$ for any $\nu>0$ and $\nu \in \mathbb{R}$. In this paper, we prove a conjecture of Sondow on upper bound for Ramanujan primes. An explicit bound of Ramanujan primes is also given. The proof uses explicit bounds of prime $\pi$ and $\theta$ functions due to Dusart.
\end{abstract}

\section{INTRODUCTION}

In [3], J. Sondow defined Ramanujan primes and gave some conjectures on the behaviour of Ramanujan primes. For $n \geq 1$, the $n$th Ramanujan prime is defined to be the smallest positive integer $R_{n}$ with the property that if $x \geq R_{n}$, then $\pi(x)-\pi\left(\frac{x}{2}\right) \geq n$ where $\pi(\nu)$ is the number of primes not exceeding $\nu$ for any $\nu>0$ and $\nu \in \mathbb{R}$. It is easy to see that $R_{n}$ is a prime for each $n$. The first few Ramanujan primes are given by $R_{1}=2, R_{2}=11, R_{3}=17, R_{4}=29, R_{5}=41, \ldots$. Sondow showed that for every $\epsilon>0$, there exists $\mathcal{N}_{0}(\epsilon)$ such that $R_{n}<(2+\epsilon) n \log n$ for $n \geq \mathcal{N}_{0}(\epsilon)$. In this note, an explicit value of $\mathcal{N}_{0}(\epsilon)$ for each $\epsilon>0$ is given. We prove

Theorem 1. Let $\epsilon>0$. For $\epsilon \leq 1.08$, let $\mathcal{N}_{0}=\mathcal{N}_{0}(\epsilon)=\exp \left(\frac{c}{\epsilon} \log \frac{2}{\epsilon}\right)$ where $c$ is given by the following table.

\begin{tabular}{|c|c|c|c|c|c|c|}
\hline$\epsilon \in$ & $\left(0, \frac{2}{11}\right]$ & $\left(\frac{2}{11}, .4\right]$ & $(.4, .6]$ & $(.6, .8]$ & $(.8,1]$ & $(1,1.08]$ \\
\hline$c$ & 4 & 5 & 6 & 7 & 8 & 9 \\
\hline
\end{tabular}

For $\epsilon>1.08$, let $\mathcal{N}_{0}=\mathcal{N}_{0}(\epsilon)$ be given by

\begin{tabular}{|c|c|c|c|c|c|c|}
\hline$\epsilon \in$ & $(1.08,1.1]$ & $(1.1,1.21]$ & $(1.21,1.3]$ & $(1.3,2.5]$ & $(2.5,6]$ & $(6, \infty)$ \\
\hline $\mathcal{N}_{0}$ & 169 & 101 & 74 & 48 & 6 & 2 \\
\hline
\end{tabular}

Then

$$
R_{n}<(2+\epsilon) n \log n \text { for } n \geq \mathcal{N}_{0}(\epsilon) .
$$

Sondow also showed that $p_{2 n}<R_{n}<p_{4 n}$ for $n>1$ and he conjectured ([3, Conjecture 1]) that $R_{n}<p_{3 n}$ for all $n \geq 1$, where $p_{i}$ is the $i$ th prime number. We derive the assertion of conjecture as a consequence of Theorem 1 . We have

Theorem 2. For $n>1$, we have

$$
p_{2 n}<R_{n}<p_{3 n} .
$$

1991 Mathematics Subject Classification. 11A41.

Key words and phrases. Ramanujan primes; primes.

Appeared in International Journal of Number Theory, Vol. 6, No. 8 (2010) 1869-1873. 
We prove Theorems 1 and 2 in Section 3. In Section 2, we give preliminaries and lemmas for the proof which depend on explicit and sharp estimates from prime number theory.

\section{Lemmas}

We begin with the following estimates from prime number theory. Recall that $p_{i}$ is the $i$ th prime prime and $\pi(\nu)$ is the number of primes $\leq \nu$. Let $\theta(\nu)=\sum_{p \leq \nu} \log p$ where $p$ is a prime.

Lemma 2.1. For $\nu \in \mathbb{R}$ and $\nu>1$, we have

(a) $p_{i}>i \log i$ for $i \geq 1, i \in \mathbb{Z}$.

(b) $\nu\left(1-\frac{0.006788}{\log \nu}\right) \leq \theta(\nu) \leq \nu\left(1+\frac{0.006788}{\log \nu}\right)$ for $\nu \geq 10544111$.

(c) $\frac{\nu}{\log \nu-1} \underset{\nu \geq 5393}{\leq} \pi(\nu) \underset{\nu>1}{\leq} \frac{\nu}{\log \nu}\left(1+\frac{1.2762}{\log \nu}\right)$.

The estimate $(a)$ is due to Rosser [2] and the estimates $(b)$ and $(c)$ are due to Dusart $[1$, p. 54].

From Lemma $2.1(b)$ and $(c)$, we obtain

Lemma 2.2. Hence for $x \geq 2 \cdot 10544111$, we obtain

$$
\pi(x)-\pi\left(\frac{x}{2}\right) \geq \frac{x}{2 \log x}\left(1-\frac{0.020364}{\log x}\right)=: F(x) \text { for } x \geq 2 \cdot 10544111
$$

and

$$
\pi(x)-\pi\left(\frac{x}{2}\right) \geq \frac{x}{2(\log x-1)}\left\{1-\frac{1}{\log \frac{x}{2}}\left(\delta_{1}-\frac{\delta_{2}}{\log \frac{x}{2}}\right)\right\}=: F_{1}(x) \text { for } x \geq 5393
$$

where $\delta_{1}=.2762+\log 2$ and $\delta_{2}=1.2762(1-\log 2)$.

Proof. For $x \geq 2 \cdot 10544111$, we obtain from Lemma 2.1 (b) that

$$
\begin{aligned}
\pi(x)-\pi\left(\frac{x}{2}\right) & \geq \frac{\theta(x)-\theta\left(\frac{x}{2}\right)}{\log x} \\
& \geq \frac{x\left(1-\frac{0.006788}{\log x}\right)-\frac{x}{2}\left(1+\frac{0.006788}{\log \frac{x}{2}}\right)}{\log x} \\
& =\frac{x}{2 \log x}\left(1-\frac{0.006788}{\log x}\left(2+\frac{\log x}{\log \frac{x}{2}}\right)\right) \\
& \geq \frac{x}{2 \log x}\left(1-\frac{0.006788}{\log x}(2+1)\right)
\end{aligned}
$$

which imply (1). For $x \geq 5393$, we have from Lemma 2.1 (c) that

$$
\begin{aligned}
\pi(x)-\pi\left(\frac{x}{2}\right) & \geq \frac{x}{\log x-1}-\frac{\frac{x}{2}}{\log \frac{x}{2}}\left(1+\frac{1.2762}{\log \frac{x}{2}}\right) \\
& =\frac{x}{2(\log x-1)}\left\{2-\left(1+\frac{\log 2-1}{\log \frac{x}{2}}\right)\left(1+\frac{1.2762}{\log \frac{x}{2}}\right)\right\} \\
& \geq \frac{x}{2(\log x-1)}\left\{1-\frac{1}{\log \frac{x}{2}}\left(\delta_{1}-\frac{\delta_{2}}{\log \frac{x}{2}}\right)\right\}
\end{aligned}
$$

implying (2).

For the proof of Theorem 1 for $\epsilon \leq .4$, we shall use the inequality (1). Then we may assume $n \leq \mathcal{N}_{0}(.4)$ for $\epsilon>.4$ and we use (2) to prove the assertion. 


\section{Proof of Theorems 1 And 2}

For simplicity, we write $\epsilon_{1}=\frac{\epsilon}{2}, \log _{2} n:=\log \log n$ and

$$
f_{0}(n):=\log n+\log _{2} n+\log \left(1+\epsilon_{1}\right) \text { and } f_{1}(n):=\frac{\log _{2} n+\log \left(2+2 \epsilon_{1}\right)}{\log n} .
$$

Let $x \geq\left(2+2 \epsilon_{1}\right) n \log n$ with $n \geq \mathcal{N}_{0}(\epsilon)=\exp \left(\frac{c}{2 \epsilon_{1}} \log \frac{1}{\epsilon_{1}}\right)=: n_{0}\left(\epsilon_{1}\right)$. Then $\log x \geq f_{0}(n)+\log 2$ for $n \geq n_{0}\left(\epsilon_{1}\right)$.

First we consider $\epsilon_{1} \leq .2$. We observe that $F(x)$ is an increasing function of $x$ and $2 n_{0}(.2) \log \left(n_{0}(.2)\right)>$ $2 \cdot 10544111$. Therefore we have from (1) that

$$
\frac{\pi(x)-\pi\left(\frac{x}{2}\right)}{n} \geq \frac{1+\epsilon_{1}}{1+f_{1}(n)}\left(1-\frac{0.020364}{f_{0}(n)+\log 2}\right)=: G(n) .
$$

$G(n)$ is again an increasing function of $n$. If $G\left(n_{0}\left(\epsilon_{1}\right)\right)>1$, then $\pi(x)-\pi\left(\frac{x}{2}\right)>n$ for all $x \geq(2+$ $\left.2 \epsilon_{1}\right) n \log n$ when $n \geq n_{0}\left(\epsilon_{1}\right)$ and hence $R_{n}<\left(2+2 \epsilon_{1}\right) n \log n$ for $n \geq n_{0}\left(\epsilon_{1}\right)$. Therefore we show that $G\left(n_{0}\right)>1$. It suffices to show

$$
\epsilon_{1}-\frac{0.020364\left(1+\epsilon_{1}\right)}{f_{0}(n)+\log 2}>f_{1}(n)=\frac{\log _{2} n_{0}+\log \left(2+2 \epsilon_{1}\right)}{\log n_{0}}
$$

for which it is enough to show

$$
\epsilon_{1} \geq \frac{\log _{2} n_{0}+\log \left(2+2 \epsilon_{1}\right)+0.020364\left(1+\epsilon_{1}\right)}{\log n_{0}} .
$$

Since $\log n_{0}=\frac{c}{2 \epsilon_{1}} \log \frac{1}{\epsilon_{1}}=\frac{c_{1}}{\epsilon_{1}} \log \frac{1}{\epsilon_{1}}$ with $c_{1}=2,2.5$ when $\epsilon_{1} \leq \frac{1}{11}, \frac{1}{5}$, respectively, we need to show

$$
\frac{\left(c_{1}-1\right) \log \frac{1}{\epsilon_{1}}}{\log _{2} \frac{1}{\epsilon_{1}}+\log c_{1}+\log \left(2+2 \epsilon_{1}\right)+0.020364\left(1+\epsilon_{1}\right)} \geq 1 .
$$

The left hand side of the above expression is an increasing function of $\frac{1}{\epsilon_{1}}$ and the inequality is valid at $\frac{1}{\epsilon_{1}}=11,5$ implying the assertion for $\epsilon_{1} \leq .2$.

Thus we now take $.2<\epsilon_{1} \leq .49$. We may assume that $n<n_{0}(.2)$. Since $x \geq\left(2+2 \epsilon_{1}\right) n_{0} \log n_{0}>5393$, we have from (2) that

$$
\frac{\pi(x)-\pi\left(\frac{x}{2}\right)}{n} \geq \frac{1+\epsilon_{1}}{1+f_{1}(n)-\frac{1}{\log n}}\left\{1-\frac{1}{f_{0}(n)}\left(\delta_{1}-\frac{\delta_{2}}{f_{0}(n)}\right)\right\} .
$$

Note that the right hand side of the above inequality is an increasing function of $n$ since $n<n_{0}(.2)$. We show that the right hand side of the above inequality is $>1$. Since $n \geq n_{0}\left(\epsilon_{1}\right)$, it suffices to show

$$
\begin{aligned}
& \log n_{0}\left(\epsilon_{1}+\frac{1}{\log n_{0}}-f_{1}\left(n_{0}\right)\right)-\frac{1+\epsilon_{1}}{\frac{f_{0}\left(n_{0}\right)}{\log n_{0}}}\left(\delta_{1}-\frac{\delta_{2}}{f_{0}\left(n_{0}\right)}\right) \\
= & \epsilon_{1} \log n_{0}+1-\log _{2} n_{0}-\log \left(2+2 \epsilon_{1}\right)-\frac{1+\epsilon_{1}}{1+f_{1}\left(n_{0}\right)-\frac{\log 2}{\log n_{0}}}\left(\delta_{1}-\frac{\delta_{2}}{f_{0}\left(n_{0}\right)}\right)
\end{aligned}
$$

is $>0$. Since $n_{0}\left(\epsilon_{1}\right)=\exp \left(\frac{c_{1}}{\epsilon_{1}} \log \frac{1}{\epsilon_{1}}\right)$ where $c_{1}=3,3.5,4$ if $.2<\epsilon_{1} \leq .3, .3<\epsilon_{1} \leq .4$ and $.4<\epsilon_{1} \leq .49$, respectively, we observe that the right hand side of the above equality is equal to

$$
\left(c_{1}-1\right) \log \frac{1}{\epsilon_{1}}+1-\log _{2} \frac{1}{\epsilon_{1}}-\log \left(2 c_{1}+2 c_{1} \epsilon_{1}\right)-\frac{1+\epsilon_{1}}{1+f_{1}\left(n_{0}\right)-\frac{\log 2}{\log n_{0}}}\left(\delta_{1}-\frac{\delta_{2}}{f_{0}\left(n_{0}\right)}\right)
$$


This is an increasing function of $\frac{1}{\epsilon_{1}}$. We find that the above function is $>0$ for $\epsilon_{1} \in\{.3, .4, .49\}$ implying $R_{n}<\left(2+2 \epsilon_{1}\right) n \log n$ for $n \geq n_{0}\left(\epsilon_{1}\right)$ when $\epsilon_{1} \leq .49$. Further we observe that $n_{0}(.49) \leq 339$. As a consequence, we have

$$
R_{n}<2.98 n \log n \text { for } n \geq 339 \text {. }
$$

and

$$
\pi(x)-\pi\left(\frac{x}{2}\right) \geq 339 \text { for } x \geq 2.98 \cdot 339 \log 339>5885 .
$$

Let $n<339$. We now compute $R_{n}$ by computing $\pi(x)-\pi\left(\frac{x}{2}\right)$ for $p_{2 n}<x \leq 5885$. Recall that $R_{n}>p_{2 n}$ for $n>1$. We find that $\frac{R_{n}}{n \log n}<2.98,3,3.05,3.08$ for $n \geq 220,219,171,169$, respectively. Clearly $\frac{R_{n}}{n \log n}<2+\epsilon$ for $n \geq \mathcal{N}_{0}(\epsilon)$ when $\epsilon \leq 1.08$. Thus $R_{n}<3 n \log n$ for $n \geq 219$ and $R_{n}<3.08 n \log n$ for $n \geq 169$. For $\epsilon>1.08$, we check that the assertion is true by computing $R_{n}$ for each $n<169$. This proves Theorem 1 .

Now we derive Theorem 2. From the above paragraph, we obtain $R_{n}<3 n \log n$ for $n \geq 219$. By Lemma $2.1(a)$, we have $p_{3 n}>3 n \log 3 n$ for all $n \geq 1$ implying the assertion of Theorem 2 for $n \geq 219$. For $n<219$, we check that $R_{n}<p_{3 n}$ and Theorem 2 follows.

\section{ACKNOWLEDGMENTS}

I thank Professor Cam Stewart for his encouragement and NSERC for support towards attending CANT 2009 at New York where a part of this work was done. I also thank Professor Jonathan Sondow for sharing his preprint with me and for his comments on an earlier draft of the paper. I would like to thank an anonymous referee for his remarks on an earlier version of the paper.

\section{REFERENCES}

[1] P. Dusart, Inégalitiés explicites pour $\psi(X), \theta(X), \pi(X)$ et les nombres premiers, C. R. Math. Rep. Acad. Sci. Canada 21(1) (1999), 53-59.

[2] B. Rosser, The $n$-th prime is greater than $n \log n$, Proc. London Math. Soc. 45 (1938), 21-44.

[3] J. Sondow, Ramanujan primes and Bertrand's postulate, Amer. Math. Monthly 116 (2009), 630-635.

E-mail address: shantalaishram@gmail.com, shanta@isid.ac.in

Stat-Math Unit, Indian Statistical Institute(ISI), 7 SJS Sansanwal Marg, New Delhi 110016, India 